\title{
Right upper lobe tracheal bronchus: anesthetic challenge in one-lung ventilated patients -A report of three cases-
}

\author{
Dong Kyu Lee, Young Min Kim, Hee Zoo Kim, and Sang Ho Lim \\ Department of Anesthesiology and Pain Medicine, Korea University Guro Hospital, Seoul, Korea
}

Tracheal bronchus (TB) is an aberrant, accessary or ectopic bronchus arising almost exclusively from the right side of the tracheal wall above the carina. In our center, 673 bronchoscopic examinations were performed from 2009 to 2011 in patients undergoing one lung ventilation (OLV) and 3 TB were found. The incidence of a TB at bronchoscopy was $0.45 \%$ in our research, which is consistent with the reported incidence range from $0.1-5 \%$. The clinician should consider the possibility of anomalous right upper lobe bronchus and perform bronchoscopy prior to the right bronchial blocker insertion, when left-sided OLV using bronchial blocker is planned. Also, for the patient with $\mathrm{TB}$, a double lumen tube insertion is recommended than a blocker insertion to achieve OLV completely. (Korean J Anesthesiol 2013; 64: 448-450)

Key Words: Bronchial blocker, Double lumen tube, One lung ventilation, Tracheal bronchus.

Tracheal bronchus (TB), an aberrant, accessary or ectopic bronchus arising almost exclusively from the right side of tracheal wall above the carina, has been reported to occur approximately in $0.1-5 \%$ of the population, which depends on the diagnostic means [1]. In our center, 673 routine bronchoscopies were studied (2009-2011) in patients undergoing one lung ventilation (OLV) and 3 TB were found. We present 3 cases with $\mathrm{TB}$ that makes it impossible to isolate the right lung with a bronchial blocker (BB).

\section{Case Reports}

\section{Case 1}

A 77-year-old woman, with the weight of $63 \mathrm{~kg}$, height of 153 $\mathrm{cm}$, and classified as the American Society of Anesthesiologists (ASA) physical classification status II with carcinoma of the esophagus was scheduled for a subtotal esophagectomy, through a right thoracotomy approach. She had undergone a plication of the diaphragm for diaphragmatic eventration caused by phrenic nerve palsy through the same approach,

Received: June 14, 2012. Revised: July 13, 2012. Accepted: August 6, 2012.

Corresponding author: Dong Kyu Lee, M.D., Ph.D., Department of Anesthesiology and Pain Medicine, Korea University Guro Hospital, 97, Gurodong-gil, Guro-gu, Seoul 152-703, Korea. Tel: 82-2-2626-1437, Fax: 82-2-851-9897, E-mail: entopic@naver.com

(c) This is an open-access article distributed under the terms of the Creative Commons Attribution Non-Commercial License (http:// creativecommons.org/licenses/by-nc/3.0/), which permits unrestricted non-commercial use, distribution, and reproduction in any medium, provided the original work is properly cited. 
8 years before in our institution. The record showed a leftsided, $35 \mathrm{~F}$ double lumen tube (DLT) was used and no specific event was recorded. We used a BB (Coopdech ${ }^{\circledR}$, Daiken medical, Osaka, Japan) to perform the left-sided OLV. A $2.2 \mathrm{~mm}$ fiberoptic bronchoscope (LF-P, Olympus, Tokyo, Japan) was advanced into the 7.0 single-lumen endotracheal tube (SLT), and confirmed the right positioning of the $\mathrm{BB}$ in the right main bronchus. Then, the balloon inflated with adequate volume of air. Unfortunately, we did not perform an auscultation of the chest, because we assured successful OLV based on the bronchoscopic finding. At the beginning of a surgery, blocker failed to provide satisfactory right upper lobe (RUL) collapse. A careful bronchoscopic examination revealed the presence of a smaller opening superior-lateral to blocker cuff. We replaced the BB with a left-sided, 35 F DLT (Mallinckrodt ${ }^{\mathrm{TM}}$, Covidien, Irland). Subsequent bronchoscopy showed the presence of the anomalous $\mathrm{TB}$, approximately $2 \mathrm{~cm}$ proximal to the carina. Thereafter, routine bronchoscopy was performed prior to OLV to identify anatomic variants in our center.

\section{Case 2}

A 69 -year-old woman, $68 \mathrm{~kg}, 147 \mathrm{~cm}$, ASA physical classification status II with lung cancer, was scheduled to undergo a RUL lobectomy through a right thoracotomy approach. Leftsided OLV, using a 7.0 SLT with BB was planned. A fiberoptic bronchoscope performed prior to the insertion of a blocker showed a small RUL bronchus at the same level of the carina. It was thought that a blocker could simultaneously block the right main bronchus and RUL bronchus because the distance between them was short enough; so the blocker was tried first. After the balloon inflation with adequate volume of air, small opening proximal to the blocker cuff was still sighted under fiberoptic guidance. That opening resulted in continued ventilation of the RUL, despite the right positioning of the blocker. The blocker was withdrawn, then a left-sided, 35 F DLT was inserted to achieve complete right lung collapse.

\section{Case 3}

A 64-year-old man, $67 \mathrm{~kg}, 163 \mathrm{~cm}$, ASA physical classification status II with esophageal cancer, was scheduled for a esophagogastrectomy, through a right thoracotomy approach. The preoperative CT examination revealed a displaced right upper lobe bronchus, approximately $1 \mathrm{~cm}$ above the carina (Fig. 1A). Left-sided OLV, using a 37 F DLT, instead of a blocker, was planned. After anesthetic induction, a left-sided DLT was placed. A fiberoptic bronchoscope was then passed down the tube and confirmed the presence of a RUL bronchus, located in an aforementioned site (Fig. 1B). The right lung isolation was satisfactory and the case proceeded uneventfully.

\section{Discussion}

OLV is often performed for thoracic surgery, especially video assisted thoracoscopic surgery (VATS), which is a widely used surgical modality. Although DLT is the most common device for lung isolation, bronchial blockade technology is increasing. The use of BB is advantageous over the DLT in difficult intubation situations, in small adults or children, and eliminates the need for a tube exchange when postoperative mechanical ventilation is essential [2]. However, in cases with an undiagnosed TB, there are several reported cases that are unable to isolate the right lung with $\mathrm{BB}[3,4]$. Using a left-sided DLT made it possible to perform complete OLV in our cases after failing to collapse the right lung, using BB. Typically, patients with TB can be well managed with a left DLT $[3,4]$. If a left DLT is not adequate, other potential solutions, including the use a Fogarty catheter to block the TB in addition to a Univent tube [5], only a Univent tube or one BB blocking of a TB [6-8] or the use a Fogarty catheter in the TB with BB located in the right main bronchus [8]. The normal RUL bronchus arises from $1-3 \mathrm{~cm}$, distal to the carina; otherwise, TB is usually $2 \mathrm{~cm}$ above the carina [1]. TB may be displaced or supernumerary (Fig. 2). A displaced TB is defined when the normal RUL bronchus or segment is transposed
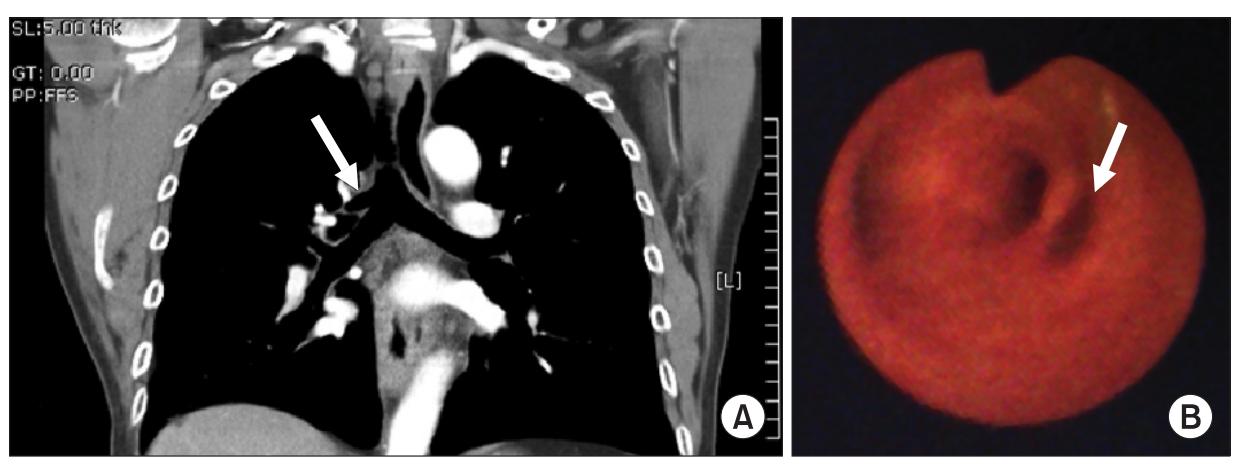

Fig. 1. (A) Coronal CT imaging demonstrates a tracheal bronchus (arrow) approximately $1 \mathrm{~cm}$ proximal to the carina. (B) Fiberoptic examination of the distal trachea confirms a tracheal bronchus (arrow). 
A

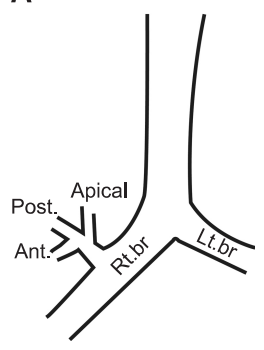

D

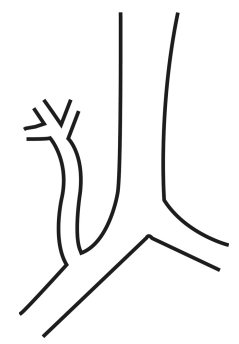

B

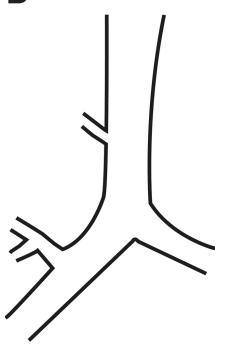

$E$

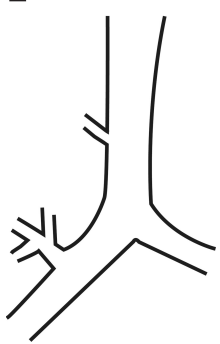

C

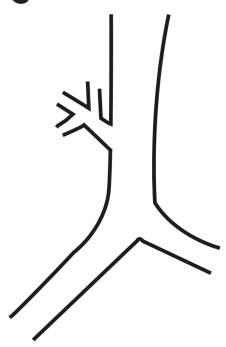

F

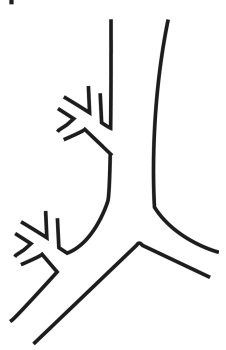

Fig. 2. Displaced (B, C) and supernumerary (E, F) tracheal bronchi. They are determined based on the presence or absence of normal trifurcation of the RUL bronchus [9]. (A) Normal, (B) Displaced apical bronchus, (C) Displaced lobar bronchus, (D) Aberrant lobar bronchus, (E) Supernumerary apical bronchus, (F) Supernumerary lobar bronchus. Rt. br: right main stem bronchus, Lt. br: left main bronchus, Ant: anterior segment, Post: posterior segment, Apical: apical segment.

from the right main bronchus to the trachea. There is no actual RUL bronchus coming from the right main bronchus. Supernumerary TB is the accessory segments or bronchi when there is a normal trifurcation of the RUL bronchus [9]. This congenital anomaly is frequently regarded as an incidental finding during bronchoscopy or radiologic examination, but it is occasionally contributing to the respiratory disease, such as pneumonia [1,9]. According to previous reports, although, most of the tracheobronchial tree anomalies are well diagnosed on a conventional chest CT [10], there are inherent limitations of conventional CT for displaying airways anatomy [11]. Multidetector CT scanner and computerized reconstruction methods can help to overcome these limitations, but higher quality 2D and 3D reformation images are not conventionally done [11]. A coronal CT scan identified a TB only in the third case; thus, fiberoptic bronchoscopy played a crucial role in the detection of TB [12]. We reported that the incidence of TB is $0.45 \%$ at bronchoscopy. This figure agrees with the previously

reported incidence, which ranges between 0.1 and 5\% [1]. Even though the incidence of TB is low, careful bronchoscopic examination is highly recommended before right the insertion of $\mathrm{BB}$ to detect any tracheobroncheal anomaly when left-sided OLV using BB is planned. Further, when a TB is found, DLT insertion is recommended than that of blocker insertion to achieve OLV completely.

\section{References}

1. Barat M, Konrad HR. Tracheal bronchus. Am J Otolaryngol 1987; 8: 118-22.

2. Narayanaswamy M, McRae K, Slinger P, Dugas G, Kanellakos GW, Roscoe A, et al. Choosing a lung isolation device for thoracic surgery: a randomized trial of three bronchial blockers versus double-lumen tubes. Anesth Analg 2009; 108: 1097-101.

3. Kim YS, Kim DW, Cho DG. Failure to collapse right lung using a single lumen tube with bronchial blocker in a patient with congenital right tracheal bronchus. Korean J Anesthesiol 2001; 40: 829-32.

4. Peragallo RA, Swenson JD. Congenital tracheal bronchus: the inability to isolate the right lung with a univent bronchial blocker tube. Anesth Analg 2000; 91: 300-1.

5. Lee HL, Ho AC, Cheng RK, Shyr MH. Successful one-lung ventilation in a patient with aberrant tracheal bronchus. Anesth Analg 2002; 95: 492-3.

6. Toyoyama H, Minami W, Toyoda Y. Possible right lung isolation by blocking the tracheal bronchus with only a Univent tube for some patients. Anesth Analg 2003; 96: 1239.

7. Kin N, Tarui K, Hanaoka K. Successful lung isolation with one bronchial blocker in a patient with tracheal bronchus. Anesth Analg 2004; 98: 270.

8. Wiser SH, Hartigan PM. Challenging lung isolation secondary to aberrant tracheobronchial anatomy. Anesth Analg 2011; 112: 68892.

9. McLaughlin FJ, Strieder DJ, Harris GB, Vawter GP, Eraklis AJ. Tracheal bronchus: association with respiratory morbidity in childhood. J Pediatr 1985; 106: 751-5.

10. Newell JD, Thomas HM, Maurer JW. Computed tomographic demonstration of displaced right upper lobe bronchus in an adult woman with congenital heart disease. J Comput Tomogr 1984; 8: 75-9.

11. Salvolini L, Bichi Secchi E, Costarelli L, De Nicola M. Clinical applications of 2D and 3D CT imaging of the airways-a review. Eur J Radiol. 2000; 34: 9-25.

12. Zheng YJ, Deng JK, Zhang DZ, Gen YG. Diagnosis of tracheal bronchus in children. World J Pediatr 2007; 3: 286-9. 